\title{
Structure of the edge density pedestal in tokamaks
}

\author{
Weston M. Stacey \\ Fusion Research Center \\ Georgia Institute of Technology \\ January, 2004
}

\begin{abstract}
A 'first-principles' model for the structure of the edge density pedestal in tokamaks between or in the absence of edge localized magnetohyrodynamic instabilities is derived from ion momentum and particle conservation and from the transport theory of recycling neutral atoms. A calculation for (high) H-mode tokamak discharge parameters indicates that the equations have a self-consistent solution which has an edge pedestal in the ion density profile and sharp negative spikes in the poloidal velocity and radial electric field profiles in the edge pedestal, features characteristic of H-mode edge profiles. These sharp negative spikes in radial electric field and poloidal rotation produce a peak in the inward ion pinch velocity in the sharp gradient (pedestal) region which produces an edge particle transport barrier. The calculated magnitude of the density at the top of the pedestal and the density gradient scale length and radial electric field in the pedestal region are comparable to measured values.
\end{abstract}

PACS 52.55.Fi, 52.55.Vy 


\section{INTRODUCTION}

The importance of the edge pedestal in determining the enhanced performance of $\mathrm{H}$ mode (high-mode) tokamak plasmas is now widely recognized. A correlation between a pronounced steep-gradient region in the edge and enhanced plasma performance has been documented in numerous experimental studies. In addition, both experiments and numerical calculations using the more recent transport models indicate a stiff temperature profile in the plasma core, implying that the achievable central temperature depends directly on the edge pedestal temperature.

Despite more than a decade of research (see Ref. 1 for a review), the understanding of the physics that determines the structure of the edge temperature and density pedestals (i.e. the steepness of the gradients and the widths over which the steep gradients extend) is still incomplete. Although correlations (e.g. Refs. 2-6) of the experimental edge pedestal database have identified a number of apparent relationships, a first-principles prediction of edge density and temperature gradients and widths remains elusive.

The coincidence of the steep edge gradients in density and temperature with regions of strongly sheared poloidal rotation and radial electric field hints that the physics that determines the structure of the edge pedestal is complex. A number of possible physics mechanisms have been suggested.

Previously, the pressure gradient limit for the onset of ideal, infinite-n MHD ballooning modes was thought to constrain the maximum edge pressure gradient, and measured edge pressure gradients were observed to be roughly in agreement with this prediction in a number of tokamak discharges. However, in recent years many discharges with edge pressure gradients several times larger than the ideal ballooning mode limit have been observed. These observations have prompted more sophisticated MHD analyses which predict the possibility of accessing a second stability regime in which the edge pressure gradient can be much larger than the infinite-n ideal ballooning mode value without the onset of instability. There is strong experimental evidence that the dominant MHD instabilities that limit the edge pressure gradient are the intermediate-n peeling-ballooning modes driven by the edge pressure gradient and the associated bootstrap current (e.g. Refs. 7-11). It has also been proposed (e.g. Ref. 12) that a MHD stability limit on the pressure, instead of the pressure gradient, limits the pressure in the 
edge pedestal. Since a MHD stability pressure gradient or pressure limit imposes an upper limit on the pressure gradient or pressure but can not, in itself, determine the individual temperature and density gradients nor the corresponding widths of the strong gradient regions, other mechanisms have been investigated.

The observation that the sharp density buildup in the edge occurs over a distance that is comparable to the ionization mean-free-path has led to the hypothesis that the structure of the density profile in the edge is determined by neutral penetration (e.g. Refs. 13-16). However, this density buildup distance is also comparable to the ion poloidal gyroradius, which has led to the prediction that the density pedestal width in the edge is determined by orbit loss (e.g. Ref. 17).

It has been proposed (e.g. Ref. 18) that the heat conduction relation determines the local temperature gradient in the edge in terms of the conductive heat flux passing through the edge and the local value of thermal conductivity. This relation has been used to infer values for the thermal conductivity in the plasma edge (e.g. Refs. 16, 19). Similarly, it has been proposed that the local density gradient in the edge pedestal is determined by the diffusive particle flux passing through the edge and the local values of the particle diffusivity and the convective "pinch" velocity ${ }^{18}$.

A "framework" for incorporating these various physics mechanisms in a predictive model for the temperature and density gradients and widths in the plasma edge has been proposed ${ }^{16}$. However, a better understanding of the basic physics mechanisms that determine the structure of the edge pedestal is required before such a predictive model can be fully implemented.

The purpose of this paper is to obtain a better understanding of some of these basic physics mechanisms by investigating the extent to which the structure and other features of the edge density pedestal can be accounted for by 1) the requirements of plasma ion particle and momentum balance and 2) the penetration of recycling neutrals. We consider the steady-state balance equations and do not take into account the effect of ELMs on particle or momentum loss, so the analysis is applicable to discharges between or in the absence of ELMs.

We have previously developed from plasma ion particle and momentum balance a firstprinciples "diffusive-pinch" expression for the radial ion particle flux in the plasma edge in which: 1) the diffusion coefficients depend on interspecies collisional momentum exchange frequencies, cross-field momentum transport frequencies and the charge-exchange, elastic scattering and ionization frequencies that determine momentum exchange between ions and 
neutrals; and 2) the pinch velocity is specifically identified as a collection of terms depending on poloidal rotation velocities, the radial electric field, and the toroidal components of any momentum input source and induced electric field ${ }^{20,21}$. We have also previously developed, from momentum and particle balance, a model for calculating the poloidal rotation velocities and the density asymmetries over the flux surface that are needed to evaluate the neoclassical gyroviscous cross-field momentum transport frequencies ${ }^{22}$, and we have found that these gyroviscous frequencies agree in magnitude with measured momentum transport frequencies in the edge of several DIII-D discharges ${ }^{20}$. In the present paper, we combine these two calculation models to investigate the role of particle and momentum balance in determining the structure of the edge density pedestal.

In order to investigate the effect of neutral penetration on the structure of the edge density pedestal, we solve simultaneously for the transport of neutrals into the plasma and for the ion density profile in the plasma edge. The calculation model described in the previous paragraph is used to solve for the ion density profile in the edge plasma, taking into account the radial electric field and poloidal rotation velocity profiles that determine the pinch velocity profile and the ionization particle source and the momentum sink due to recycling neutrals. The transport of recycling neutrals across the separatrix and inward into the edge plasma is calculated using the "interface current balance" formulation of integral neutral particle transport theory ${ }^{23}$.

The boundary conditions for these edge profile calculations are: 1) the inward neutral flux incident across the separatrix, which is obtained from a $2 \mathrm{D}$ calculation of neutral recycling from the divertor; 2) the net outward ion flux across the separatrix, which is obtain from a particle balance on the core plasma; and 3) the ion density at the separatrix, which is obtained from a "2-point" divertor model calculation. These three "boundary condition calculations" are iterated to consistency, as described in Refs. 24.

The outline of the paper is as follows. A self-consistent model for the calculation of the plasma ion and neutral atom profiles, the radial electric field and poloidal velocity profiles, and associated quantities is summarized in section II. Results of a calculation are presented in section III to illustrate the qualitative features of the solutions and to examine the physics that determines these features of the edge density pedestal. Finally, the implications of the results are discussed in section IV. 


\section{DENSITY PEDESTAL CALCULATION MODEL}

\section{A. Generalized 'Pinch-Diffusion' Particle Flux Relations}

The particle continuity equation for ion species ' $\mathrm{j}$ ' is

$$
\nabla \cdot n_{j} v_{j}=S_{j}
$$

where $S_{j}(r, \theta)=n_{e}(r, \theta) n_{j 0}(r, \theta)\langle\sigma v\rangle_{i o n} \equiv n_{e}(r, \theta) v_{i o n}(r, \theta)$ is the ionization source rate of ion species ' $\mathrm{j}$ ' and $\mathrm{n}_{\mathrm{j} 0}$ is the local concentration of neutrals of species ' $\mathrm{j}$ '. Taking the flux surface average of this equation yields $\left\langle\left(\nabla \cdot n_{j} v_{j}\right)_{r}\right\rangle=\left\langle S_{j}\right\rangle$ because $\left.\left\langle\nabla \cdot n_{j} v_{j}\right)_{\theta}\right\rangle=0$ identically and $\left.\left\langle\nabla \cdot n_{j} v_{j}\right)_{\phi}\right\rangle=0$ by axisymmetry, which allows Eq. (1) to be written

$$
\left(\nabla \cdot n_{j} v_{j}\right)_{\theta}=S_{j}-\left\langle S_{j}\right\rangle \equiv \tilde{S}_{j}
$$

Integration of this equation, in toroidal $(r, \theta, \phi)$ coordinates, yields

$$
n_{j} v_{\theta j}=\frac{K, \bar{B}_{\theta}+r \bar{B}_{\theta} \int_{o}^{\theta}(1+\varepsilon \cos \theta) \tilde{S}_{j} d \theta}{1+\varepsilon \cos \theta} \equiv\left[K_{j}(r)+I_{j}(r, \theta)\right] B_{\theta}(r)
$$

where $K_{j}=\left\langle n_{j} v_{\theta j}\right\rangle / \bar{B}_{\theta} \approx \bar{n}_{j} \bar{v}_{\theta_{j}} / \bar{B}_{\theta}, \mathrm{I}_{\mathrm{j}}$ is defined by Eq. 3, and the overbar denotes the average value over the flux surface.

Subtracting $m_{j} v_{j}$ times Eq. (1) from the momentum balance equation for ion species ' $j$ ' and noting that $\left(\nabla \cdot n_{j} v_{j}\right)_{r} \square\left(\nabla \cdot n_{j} v_{j}\right)_{\theta}$ leads to

$$
n_{j} m_{j}\left(\boldsymbol{v}_{j} \cdot \nabla\right) v_{j}+\nabla p_{j}+\nabla \cdot \pi_{j}=n_{j} e_{j}\left(v_{j} \times \boldsymbol{B}\right)+n_{j} e_{j} \boldsymbol{E}+\boldsymbol{F}_{j}+\boldsymbol{M}_{j}-n_{j} m_{j} v_{a t}^{j} v_{j}-m_{j} \tilde{S}_{j} \boldsymbol{v}_{j}
$$

where $\mathbf{F}_{\mathrm{j}}$ represents the interspecies collisional friction, $\mathbf{M}_{\mathrm{j}}$ represents the external momentum input rate, and the last two terms represent the momentum loss rate due to elastic scattering and 
charge exchange with neutrals of all ion species ' $\mathrm{k}$ ' $\left[v_{a t j}=\Sigma_{k} n_{k 0}^{c}\left(\langle\sigma v\rangle_{e l}+\langle\sigma v\rangle_{c x}\right)_{j k}\right]$ and due to the introduction of ions with no net momentum via ionization of a neutral of species ' $\mathrm{j}$ '. Only the 'cold' neutrals that have not already suffered an elastic scattering or charge-exchange collision in the pedestal are included in $v_{a t j}$.

Taking the cross product B $\times$ Eq. (4) yields a 'radial' $\left(\mathbf{n}_{\mathrm{r}}\right)$ component equation

$$
\begin{aligned}
n_{j} e_{j} v_{r j} B^{2}= & B_{\theta}\left\{n_{j} m_{j}\left[\left(v_{j} \cdot \nabla\right) v_{j}\right]_{\phi}+[\nabla \cdot \pi]_{\phi}-M_{j \phi}-F_{j \phi}-n_{j} e_{j} E_{\phi}+m_{j}\left(n_{j} v_{a t j}+\tilde{S}_{j}\right) v_{\phi j}\right\}- \\
& B_{\phi}\left\{n_{j} m_{j}\left[\left(v_{j} \cdot \nabla\right) v_{j}\right]_{\theta}+[\nabla \cdot \pi]_{\theta}+\frac{\partial p_{j}}{\partial \ell_{\theta}}-M_{j \theta}-F_{j \theta}-n_{j} e_{j} E_{\theta}+m_{j}\left(n_{j} v_{a t j}+\tilde{S}_{j}\right) v_{\theta j}\right\}
\end{aligned}
$$

and a 'perpendicular' $\left(\mathrm{B}_{\phi} \mathbf{n}_{\theta}-\mathrm{B}_{\theta} \mathbf{n}_{\phi}\right)$ component equation

$$
n_{j} m_{j}\left[\left(v_{j} \cdot \nabla\right) v_{j}\right]_{r}+\left[\nabla \cdot \pi_{j}\right]_{r}+\frac{\partial p_{j}}{\partial r}=n_{j} e_{j}\left(v_{\theta j} B_{\phi}-v_{\phi j} B_{\theta}+E_{r}\right)
$$

and taking the scalar product B.Eq. (4) yields a third, independent parallel momentum balance equation

$$
\begin{aligned}
& B_{\phi}\left\{n_{j} m_{j}\left[\left(v_{j} \cdot \nabla\right) v_{j}\right]_{\phi}+[\nabla \cdot \pi]_{\phi}-M_{j \phi}-F_{j \phi}-n_{j} e_{j} E_{\phi}+m_{j}\left(n_{j} v_{a t j}+\tilde{S}_{j}\right) v_{\phi j}\right\} \\
= & -B_{\theta}\left\{n_{j} m_{j}\left[\left(v_{j} \cdot \nabla\right) v_{j}\right]_{\theta}+[\nabla \cdot \pi]_{\theta}+\frac{\partial p_{j}}{\partial \ell_{\theta}}-M_{j \theta}-F_{j \theta}-n_{j} e_{j} E_{\theta}+m_{j}\left(n_{j} v_{a t j}+\tilde{S}_{j}\right) v_{\theta j}\right\}
\end{aligned}
$$

The quantity $E_{\phi}{ }^{A}$ is the induced toroidal field due to transformer action.

Using Eq. (7) in Eq. (5), multiplying the result by R and taking the flux surface average leads to an expression for the flux surface average radial particle flux

$$
\begin{gathered}
\bar{R} \bar{B}_{\theta} e_{j}\left\langle n_{j} v_{r j}\right\rangle \square R B_{\theta} e_{j} \bar{n}_{j} \bar{v}_{r j}=\left\langle R^{2} \nabla \phi \cdot n_{j} m_{j}\left(v_{j} \cdot \nabla\right) v_{j}\right\rangle+\left\langle R^{2} \nabla \phi \cdot \nabla \cdot \pi_{j}\right\rangle- \\
\left\langle R M_{j \phi}\right\rangle-\left\langle R F_{j \phi}\right\rangle-\left\langle R n_{j} e_{j} E_{\phi}^{A}\right\rangle+\left\langle R m_{j}\left(n_{j} v_{a t j}+\tilde{S}_{j}\right) v_{\phi j}\right\rangle
\end{gathered}
$$

The first (inertial) term on the right vanishes identically. The remaining terms on the right represent the transport fluxes in response to the toroidal viscous force, the (beam) momentum input, the interspecies collisional momentum exchange, the inductive toroidal electric field, and the momentum loss due to interactions with neutral particles, respectively.

Neglecting the viscous and inertial terms in Eq. (6), using Eq. (3), and assuming that the radial electric field is electrostatic leads to an expression for the flow velocity of ion species ' $j$ ' in the flux surface 


$$
n_{j} v_{j}=\left(K_{j}+I_{j}\right) \boldsymbol{B}-\frac{1}{e_{j} B_{\theta}}\left(\frac{\partial p_{j}}{\partial r}+n_{j} e_{j} \frac{\partial \phi}{\partial r}\right) \boldsymbol{n}_{\phi}
$$

Flux surface averaging this equation yields an expression for the average toroidal rotation over the flux surface in terms of the average poloidal rotation and radial gradients of the pressure and electrostatic potential

$$
\bar{v}_{\phi j}=f_{p}^{-1} \bar{v}_{\theta_{j}}-\left(\bar{P}_{j}^{\prime}+\bar{\Phi}^{\prime}\right)
$$

where

$$
f_{p} \equiv B_{\theta} / B_{\phi}, \quad \bar{P}_{j}^{\prime} \equiv \frac{1}{\bar{n}_{j} e_{j} \bar{B}_{\theta}} \frac{\partial \bar{p}_{j}}{\partial r}, \quad \bar{\Phi}^{\prime} \equiv \frac{1}{\bar{B}_{\theta}} \frac{\partial \phi}{\partial r}=-\frac{\bar{E}_{r}}{\bar{B}_{\theta}}
$$

The particle fluxes within and across the flux surface are determined by Eqs. (9) and (8), respectively. In order to evaluate these fluxes it is necessary to specify the models for the viscosity and collisional friction, to know the constant $\mathrm{K}_{\mathrm{j}}$ (equivalently the average value of the poloidal velocity), and to know the radial electric field.

Using the Lorentz approximation for the collisional friction

$$
\boldsymbol{F}_{j}=-n_{j} m_{j} \sum_{k \neq j} v_{j k}\left(v_{j}-v_{k}\right)
$$

Eqs. (8) may be reduced to

$$
\begin{aligned}
\Gamma_{r j} \equiv \bar{n}_{j} \bar{v}_{r j}=\frac{1}{e_{j} \bar{B}_{\theta}}\left[-\left(\bar{M}_{\phi j}+\bar{n}_{j} e_{j} \bar{E}_{\phi}^{A}\right)\right. & +\bar{n}_{j} m_{j} \sum_{k \neq j} \bar{v}_{j k}\left(\bar{v}_{\phi j}-\bar{v}_{\phi k}\right) \\
& \left.+\bar{n}_{j} m_{j} \bar{v}_{d j}^{*} \bar{v}_{\phi j}\right]
\end{aligned}
$$

where the total momentum transfer, or 'drag', frequency $v_{d j} *$ is given by

$$
\bar{v}_{d j}^{*} \equiv \bar{v}_{d j}+\bar{v}_{a j j}+\bar{v}_{i o n j} \xi_{j}
$$

which consists of a cross-field viscous momentum transport frequency formally given by

$$
\bar{v}_{d j} \equiv\left\langle R^{2} \nabla \phi \cdot \nabla \cdot \pi_{j}\right\rangle / \bar{R} \bar{n}_{j} m_{j} \bar{\nu}_{\phi_{j}}
$$


and of the two atomic physics momentum loss terms discussed previously, with the neutral ionization source asymmetry characterized by

$$
\xi_{j} \equiv\left\langle R^{2} \nabla \phi \cdot m_{j} \tilde{S}_{j} v_{\phi j}\right\rangle / \bar{R} m_{j} \bar{S}_{j} \bar{\nu}_{\phi j}
$$

Writing

$$
\bar{P}_{j}^{\prime}=-\frac{T_{j}}{e_{j} B_{\theta}}\left(L_{n j}^{-1}+L_{T_{j}}^{-1}\right)
$$

the 'perpendicular' component of the momentum balance given by Eq. (10) can be used to eliminate the toroidal velocity in the 'radial' component given by Eq. (13) to obtain a generalized pinch-diffusion equation for each ion species present

$$
\Gamma_{j}=n_{j} D_{j j}\left(L_{n j}^{-1}+L_{T j}^{-1}\right)-n_{j} D_{j k}\left(L_{n k}^{-1}+L_{T k}^{-1}\right)+n_{j} v_{p j}
$$

where the diffusion coefficients are given by

$$
D_{j j} \equiv \frac{m_{j} T_{j}\left(v_{d j}^{*}+v_{j j}\right)}{\left(e_{j} B_{\theta}\right)^{2}}, D_{j k} \equiv \frac{m_{j} T_{k} v_{j k}}{e_{j} e_{k} B_{\theta}{ }^{2}}
$$

the pinch velocity is given by

$$
n_{j} v_{p j} \equiv-\frac{\bar{M}_{\phi j}}{e_{j} B_{\theta}}-\frac{n_{j} \bar{E}_{\phi}^{A}}{B_{\theta}}+\frac{n_{j} m_{j} v_{d_{j}}^{*}}{e_{j} B_{\theta}}\left(\frac{E_{r}}{B_{\theta}}\right)+\frac{n_{j} m_{j} f_{p}^{-1}}{e_{j} B_{\theta}}\left(\left(v_{j k}+v_{d j}^{*}\right) \bar{v}_{\theta j}-v_{j k} \bar{v}_{\theta k}\right)
$$

and where a sum over the ' $\mathrm{k} ' \neq \mathrm{j}$ terms is understood when more than two ion species are present. Note that the 'self-diffusion' coefficient $\mathrm{D}_{\mathrm{jj}}$ involves the atomic physics and viscous momentum transfer rates as well as the interspecies collisional momentum exchange frequency.

\section{B. Rotation Velocities and Radial Electric Field}


Taking the flux surface average of the toroidal component of Eq. (4)-the term in \{\} on the left side of Eq. (7)---yields a coupled set of equations for the toroidal velocities of the different ion species present plus the electrons

$$
\left(v_{d j}^{*}+\sum_{k \neq j} v_{j k}\right) v_{\phi j}-\sum_{k \neq j} v_{j k} v_{\phi k}=\frac{\left(n_{j} e_{j} E_{\phi}^{A}+e_{j} \Gamma_{j} B_{\theta}+M_{\phi j}\right)}{n_{j} m_{j}} \equiv y_{j}
$$

Since the condition $\left(\mathrm{n}_{\text {carbon }} \mathrm{Z}_{\text {carbon }}^{2} / \mathrm{n}_{\mathrm{e}}\right)>>\left(\left(\mathrm{m}_{\mathrm{e}} / \mathrm{m}_{\mathrm{D}}\right)^{1 / 2} \approx 0.016\right.$ is satisfied in most plasmas, the ion-electron collisions can be neglected relative to the ion-impurity collisions in Eq. (21). In the limiting case of a two-species ion-impurity (i-I) plasma, the two Eqs. (21) can be solved to obtain the toroidal rotation velocity of each species

$$
v_{\phi J}=\frac{\left[1+\left(v_{d k}^{*} / v_{k j}\right)\right] y_{j}+y_{k}}{\left[\left\{1+\left(v_{d k}^{*} / v_{k j}\right)\right\}\left\{1+\left(v_{d j}^{*} / v_{j k}\right)\right\}-1\right]}
$$

The toroidal rotation is driven by the input beam torque $\left(R M_{\varphi \mathrm{j}}\right)$, the input torque associated with the induced field $\left(R n_{j} e_{j} E_{\varphi}\right)$, and by the internal torque due to the radial ion flow $\left(e_{j} B_{\theta} \Gamma_{j}\right)$ which enter the $y_{\mathrm{j}}$, and depends on the radial transfer rate of toroidal angular momentum $\left(v_{\mathrm{dj}}{ }^{*}\right)$ due to viscous, atomic physics and convective effects and on the interspecies momentum exchange rate $\left(v_{\mathrm{jk}}\right)$.

The difference in toroidal rotation velocities of the two species is

$$
v_{\phi j}-v_{\phi k}=\frac{\left(v_{d k}^{*} / v_{k j}\right) y_{J}-\left(v_{d j}^{*} / v_{j k}\right) y_{k}}{\left[\left\{1+\left(v_{d k}^{*} / v_{k j}\right)\right\}\left\{1+\left(v_{d j}^{*} / v_{j k}\right)\right\}-1\right]}
$$

In order to actually evaluate the above equations it is necessary to specify the toroidal viscous force, $\left\langle R^{2} \nabla \phi \cdot \nabla \cdot \pi\right\rangle$, which determines the viscous momentum transport frequency, $v_{\mathrm{dj}}$, given by Eq. (15). There are three neoclassical viscosity components-parallel, perpendicular and gyroviscous. The "parallel' component of the neoclassical viscosity vanishes identically in 
the viscous force term, and the 'perpendicular' component is several orders of magnitude smaller than the 'gyroviscous' component ${ }^{25}$

$$
\left\langle R^{2} \nabla \phi \cdot \nabla \cdot \pi_{j}\right\rangle=\frac{1}{2} \tilde{\theta}_{j} G_{j} \frac{n_{j} m_{j} T_{j}}{e_{j} B_{\phi}} \frac{v_{\phi j}}{\bar{R}} \equiv R n_{j} m_{j} \nu_{d j} v_{\phi j}
$$

where

$$
\begin{gathered}
\tilde{\theta}_{j} \equiv\left(4+\tilde{n}_{j}^{c}\right) \tilde{v}_{\phi j}^{s}+\tilde{n}_{j}^{s}\left(1-\tilde{v}_{\phi j}^{c}\right) \\
=\left(4+\tilde{n}_{j}^{c}\right)\left[-\left(\hat{v}_{\theta j} / \hat{v}_{\phi j}\right)\left(\Phi^{s}+\tilde{n}_{j}^{s}\right)+\Phi^{s}\right]+\tilde{n}_{j}^{s}\left[\left(\hat{v}_{\theta j} / \hat{v}_{\phi j}\right)\left(2+\Phi^{c}+\tilde{n}_{j}^{c}\right)-\Phi^{c}\right]
\end{gathered}
$$

represents poloidal asymmetries and

$$
G_{j} \equiv-\frac{r}{\eta_{4 j} v_{\phi j}} \frac{\partial\left(\eta_{4 j} v_{\phi j}\right)}{\partial r}
$$

with the gyroviscosity coefficient $\eta_{4 j} \approx n_{j} m_{j} T_{j} / e_{j} B$ and $\mathrm{L}_{\mathrm{x}}^{-1}={ }_{-}(\mathrm{dx} / \mathrm{dr}) / \mathrm{x}$.

In order to evaluate Eq. (25) it is first necessary to calculate the sine and cosine components of the density and toroidal velocity poloidal variations over the flux surface. (The electron momentum balance can be used to relate the sine and cosine components of the potential variation to the corresponding components of the density variations.) A low-order Fourier expansion of the densities and rotation velocities over the flux surface can be made, and Eq. (9) can be used to relate the Fourier components of the rotation velocities for species ' $j$ ' to the Fourier components of the density for that species. These results then can be used in the poloidal component of Eq. (4)---the \{ \} term on the right in Eq. (7)---the flux surface average of which with $1, \sin \theta$ and $\cos \theta$ weighting then yields a coupled set of 3 nonlinear equations per species that can be solved numerically for the flux surface average poloidal velocities and the sine and cosine components of the density variations, for the various ion species present, over the flux surface $^{21}$. These equations are 


$$
\begin{aligned}
& \hat{v}_{\theta j}\left[-q \hat{v}_{\phi j} \varepsilon\left(\tilde{n}_{j}^{s}+\Phi^{s}\right)-q^{2} f_{j} f_{p}\left(1+\Phi^{c}+\frac{2}{3} \tilde{n}_{j}^{c}\right)+f_{p} \sum_{k \neq j} v_{j k}^{*}+\right. \\
& \frac{q}{\varepsilon} v_{a t j}^{*} f_{p}+\frac{1}{2} f_{p} \varepsilon v_{i o n j}^{*}\left\{\left(1+\tilde{n}_{j}^{c}\right)\left(\frac{\bar{n}_{e}}{\bar{n}_{j}}\left(\begin{array}{c}
\tilde{n}_{e}^{c}+\tilde{n}_{o j}^{c} \\
n^{\prime}
\end{array}\right)-\left(\begin{array}{c}
\tilde{n}_{j}+\tilde{n}_{o j}^{c} \\
n^{\prime}
\end{array}\right)\right)+\right. \\
& \left.\left.\tilde{n}_{j}\left(\bar{n}_{e}\left(\tilde{n}_{j}^{s}+\tilde{n}_{i j}^{s}\right)-\left(\tilde{n}_{j}^{s}+\tilde{n}_{o j}^{s}\right)\right)\right\}\right]-\sum_{k \neq j} v_{k \theta}\left[f_{p} v_{j k} \sqrt{\frac{m_{j}}{m_{k}}}\right]= \\
& -\hat{v}_{r j}-q \varepsilon \frac{1}{4} \tilde{n}_{j}^{s}-q \varepsilon \Phi_{j}\left[\frac{1}{4}\left(\Phi^{s}+\tilde{n}_{j}^{c} \Phi^{s}-\tilde{n}_{j}^{s} \Phi^{c}\right)\right]-q^{2} f_{j} f_{p}\left(\hat{v}_{\phi_{j}}+\mathscr{P}_{j}\right) \Phi^{c} \\
& -q \varepsilon \hat{v}_{\phi j}\left[\left(\hat{v}_{\phi j}+\Phi_{j}^{\prime}\right) \Phi^{s}+\frac{1}{2} \hat{v}_{\phi j} \tilde{n}_{j}^{s}\right]-\frac{\overline{n_{e}}}{\overline{n_{j}}} v_{i o n j}^{*} q\left[\hat { v } _ { \phi j } \varepsilon \left(\begin{array}{c}
\tilde{n}_{e}^{c}+\tilde{n}_{o j}^{c} \\
)
\end{array}\right.\right. \\
& \left.-\frac{2}{3} q f_{j}\left(\tilde{n}_{e}^{s}+\tilde{n}_{o j}^{s}\right)\right]
\end{aligned}
$$

$$
\begin{aligned}
& \tilde{n}_{j}\left[\frac{1}{3} \frac{q^{2}}{\varepsilon} f_{j} f_{p} \hat{v}_{\theta j}+\frac{1}{2} \varepsilon \hat{v}_{r j}-\frac{1}{2} \varepsilon f_{p} \sum_{k \neq j} v_{j k}^{*} \hat{v}_{\theta k} \sqrt{\frac{m_{j}}{m_{k}}}+\frac{1}{2} q v_{i o n}^{*} f_{p} \hat{v}_{\theta j}\right] \\
& +\tilde{n}_{j}^{c}\left[\frac{1}{2} q f_{p}^{2} \hat{v}_{\theta j}^{2}-\frac{1}{4} q+\frac{1}{2} q v_{a t j}^{*} v_{i o n j}^{*}\right]=-\frac{1}{2} \varepsilon f_{p} \sum_{k \neq j} v_{j k}^{*} \hat{v}_{\theta j} \tilde{n}_{k}^{s} \\
& -\frac{1}{4} q \Phi_{j}\left[-\overleftarrow{\Phi}^{c}\right]-\frac{q^{2}}{\varepsilon} f_{j} f_{p}\left[\frac{1}{2}\left(\hat{v}_{\theta j}-\hat{v}_{\phi j}-P_{i}^{\prime}\right) \Phi^{s}-\frac{1}{2} q f_{p}^{2} \hat{v}_{\theta j}^{2}-\frac{1}{2} q \hat{v}_{\phi j}^{2}\right. \\
& -\frac{1}{2} q v_{a t j}^{*}\left[f_{p} \hat{v}_{\theta j} \tilde{n}_{o j}^{s}+v_{i o n j}^{*} \tilde{n}_{o j}^{c}\right]-q v_{i o n j}^{*} f_{p}\left[\frac{1}{2} \hat{v}_{\theta j}\left\{\tilde{n}_{o j}^{s}\left(1+\frac{\bar{n}_{e}}{\bar{n}_{j}}\right)+\frac{\bar{n}_{e}}{\bar{n}_{j}} \tilde{n}_{e}^{s}\right\}\right. \\
& \left.+\frac{1}{3} \frac{q}{\varepsilon} f_{j} \frac{\bar{n}_{e}}{\bar{n}_{j}}\left(\begin{array}{c}
\tilde{n}_{e}^{c} \\
\tilde{n}_{e} \\
\tilde{n}_{o j}
\end{array}\right)\right]
\end{aligned}
$$

and 


$$
\begin{aligned}
& \tilde{n}_{j}^{c}\left[\frac{1}{3} \frac{q^{2}}{\varepsilon} f_{j} f_{p} \hat{v}_{\theta j}+\frac{1}{2} \varepsilon \hat{v}_{r j}-\frac{1}{2} \varepsilon f_{p} \sum_{k \neq j} v_{j k}^{*} \hat{v}_{\theta k} \sqrt{\frac{m_{j}}{m_{k}}}+\frac{1}{2} q v_{i o n j}^{*} f_{p} \hat{v}_{\theta j}\right] \\
& +\tilde{n}_{j}^{s}\left[-\frac{1}{2} q f_{p} \hat{v}_{\theta j}^{2}+\frac{1}{4} q-\frac{1}{2} q v_{a t j}^{*} v_{i o n j}^{*}\right]=-\sum_{k \neq j} \tilde{n}_{k}^{c}\left[\frac{1}{2} \varepsilon f_{p} v_{j k}^{*} \hat{v}_{\theta j}\right] \\
& -\frac{1}{4} q \Phi_{j}\left[\Phi^{s}\right]-\frac{q^{2}}{\varepsilon} f_{j} f_{p}\left[\frac{1}{2}\left\{\left(1+\Phi^{c}\right) \hat{v}_{\theta j}-\left(\hat{v}_{\phi j}-\Phi_{j}^{\prime}\right) \Phi^{c}\right\}\right] \\
& -q \hat{v}_{\phi j}^{2}\left[\frac{1}{4} \varepsilon\left\{\tilde{v}_{\phi j}^{s} \tilde{v}_{\phi j}^{c}+\tilde{n}_{j}^{c} \tilde{v}_{\phi j}^{s}+\tilde{n}_{j}^{s} \tilde{v}_{\phi j}^{c}\right\}\right]-\frac{1}{2} q v_{a l j}^{*}\left[f_{p} \hat{v}_{\theta j} \tilde{n}_{o j}^{c}-v_{i o n j}^{*} \tilde{n}_{o j}^{s}\right] \\
& -q f_{p} v_{i o n j}^{*}\left[\frac{1}{2} \hat{v}_{\theta j}\left\{\tilde{n}_{o j}^{c}\left(1+\frac{\bar{n}_{e}}{\bar{n}_{j}}\right)+\frac{\bar{n}_{e}}{\bar{n}_{j}} \eta_{e}^{b}\right\}+\frac{1}{3} \frac{q}{\varepsilon} f_{j} \frac{\bar{n}_{e}}{\bar{n}_{j}}\left(\tilde{n}_{e}^{s}+\tilde{n}_{o j}^{s}\right)\right] \\
& \hat{v}_{\theta j} \equiv \frac{\bar{v}_{\theta j}}{\left|f_{p}\right| v_{t h j}}, \quad \hat{v}_{\phi j} \equiv \frac{\bar{v}_{\phi j}}{v_{t h j}} \\
& \hat{v}_{r j} \equiv \frac{\bar{v}_{r}}{\left(\frac{m_{j} v_{t h j}}{e_{j} B_{\theta}^{0}}\right) \mid f_{p} \|\left(\frac{v_{t h j}}{q R}\right)}, \quad f_{p} \equiv \frac{B_{\theta}}{B_{\phi}}
\end{aligned}
$$

where

$$
\begin{aligned}
& \tilde{n}_{j}^{c / s} \equiv \frac{n_{j}^{c / s}}{\varepsilon}, \\
& \Phi^{c / s} \equiv \frac{\Phi^{c / s}}{\varepsilon}, \\
& \tilde{n}_{o j}^{c / s} \equiv \frac{n_{o j}^{c / s}}{\varepsilon} \\
& v_{j k}^{*} \equiv \frac{v_{j k}}{\frac{v_{t h j}}{q R}}, \\
& v_{i o n j}^{*} \equiv \frac{\bar{v}_{t a i j} r}{v_{t h j}}, \quad \quad v_{a t j}^{*} \equiv \frac{\bar{v}_{a t j} r}{v_{t h j}} \\
& \Phi_{j} \equiv \frac{e_{j} \bar{\Phi}}{T_{j}} \\
& \bigoplus_{j}^{\prime} \equiv \frac{1}{B_{\theta}^{0} \bar{n}_{j} e_{j} v_{t h j}} \frac{\partial p_{j}}{\partial r},
\end{aligned}
$$

and $f_{j}$ is defined in Eq. 33 . 
In deriving Eqs. (27)-(29), we have used the neoclassical parallel viscosity tensor obtained by extending the classical rate-of-strain tensor formalism to toroidal geometery ${ }^{25}$, leading to the poloidal component of the divergence of the parallel viscosity tensor

$$
\hat{\mathbf{n}}_{\bullet} \cdot \nabla \cdot \boldsymbol{\pi}=\eta_{o j}\left(\frac{1}{2} A_{o j}\right)\left\{\frac{1}{r} \frac{\partial \ln \left(\eta_{o j} A_{o j}\right)}{\partial \theta}-\frac{3 \sin \theta}{R}\right\}
$$

where

$$
\begin{aligned}
& \frac{1}{2} A_{o j}=\left\{-\frac{1}{3} \frac{1}{r} \frac{\partial v_{\theta j}}{\partial \theta}+v_{\theta j}\left(\frac{1}{R} \frac{1}{r} \frac{\partial R}{\partial \theta}+\frac{1}{3} \frac{1}{B_{\theta}} \frac{1}{r} \frac{\partial B_{\theta}}{\partial \theta}\right)\right. \\
& \left.+\left(\frac{B_{\theta}}{B_{\phi}}\right) \frac{R}{r} \frac{\partial\left(v_{\phi j} / R\right)}{\partial \theta}\right\} .
\end{aligned}
$$

and by replacing the classical parallel viscosity coefficient with a neoclassical form ${ }^{26}$ that takes banana-plateau collisionality effects into account.

$$
\eta_{o j}=\frac{n_{j} m_{j} v_{t h j} q R \varepsilon^{-3 / 2} v_{j j}^{*}}{\left(1+\varepsilon^{-3 / 2} v_{j j}^{*}\right)\left(1+v_{j j}^{*}\right)} \equiv n_{j} m_{j} v_{t h j} q R f_{j}\left(v_{j j}^{*}\right)
$$

The sine and cosine components of the plasma ion density asymmetries over the flux surface are indicated by $n_{j}{ }^{s}$ and $n_{j}^{c}$, respectively, and the corresponding components of the electron density asymmetries, $\mathrm{n}_{\mathrm{e}}^{\mathrm{s} / \mathrm{c}}$, are calculated from the charge neutrality requirement. The asymmetry in the recycling neutral density is likewise represented by sine and cosine components, $n_{0}{ }^{s / c}$. The electron momentum balance was used to related the sine and cosine components of the asymmetry in the electrostatic potential, $\Phi^{s / c}$, to the corresponding asymmetry in $n_{e} s / c$. For convenience, all of these asymmetry Fourier components have been divided by $\varepsilon=$ $r / R$, the local inverse aspect ratio, which is denoted by a tilde.

We note that is has been suggested ${ }^{27}$ that the above expression for the gyrovicous toroidal force underestimates the momentum transport rate in regions of steep pressure gradients and low toroidal rotation (e.g. the edge pedestal) because of failure to take into account a drift kinetic correction not present in the original Braginskii derivation. Braginskii's momentum equations 
are valid if the fluid velocities in the directions perpendicular and parallel to $\mathbf{B}$ are much larger than the diamagnetic velocity and the diagmagnetic velocity multiplied by $\mathrm{B}_{\varphi} / \mathrm{B}_{\theta}$, respectively. Ordering arguments suggest that this is not the case in the absence of a large "external" source of momentum. It is not a priori clear if the Braginskii gyroviscous formulation is correct for the conditions of the plasma edge or needs to be supplemented by a heat flux term ${ }^{28}$. In any case, the above equations have done well in predicting toroidal rotation (hence radial momentum transport) in the DIII-D core plasma ${ }^{29}$, and have predicted momentum transport frequencies in the edge pedestal of the magnitude observed in DIII-D experiments ${ }^{20}$, which motivates us to investigate their use to predict $v_{\mathrm{dj}}$ in the edge pedestal.

When Eq. (10) is used to eliminate $v_{\varphi j}$ from Eqs. (21), the resulting equations can be summed over ion species (and the toroidal electron momentum equation can be used) to obtain an explicit expression for the radial electric field

$$
\frac{E_{r}}{B_{\theta}}=\frac{\sum_{j}^{\text {ions }}\left\{M_{\phi j}+n_{j} m_{j} v_{d j}^{*}\left(P_{j}^{\prime}-f_{p}^{-1} v_{\theta j}\right)\right\}}{\sum_{j}^{i o n s} n_{j} m_{j} v_{d j}^{*}}
$$

The local electric field depends on the total local input toroidal momentum deposition $\left(M_{\varphi}=\right.$ $\left.\Sigma_{j} M_{\varphi j}\right)$, the local radial pressure gradients $\left(P_{j}{ }^{\prime}\right)$, the local poloidal velocities $\left(v_{\theta j}\right)$ and the local values of the radial momentum transfer rates $\left(v_{\mathrm{dj}}{ }^{*}\right)$ due to viscous, atomic physics and convective effects. [We note that this formulation avoids the ambiguity associated with the more common procedure of using Eq. (10) for a single ion species to determine $E_{r}$, which can result in different electric fields for different ion species.]

\section{Ion Profile in the Pedestal}

The particle flux for each ion species in the edge pedestal satisfies the balance equation

$$
\nabla \cdot \Gamma_{j} \square \frac{d \Gamma_{j}}{d x}=v_{i o n j} n_{e}=v_{i o n j}\left(\sum_{k} n_{k} Z_{k}\right)
$$


with a separatrix boundary condition $\Gamma_{\mathrm{j}}\left(\mathrm{x}_{\mathrm{sep}}\right)=\Gamma_{\text {jsep. }}$. For the main ion species, $\Gamma_{\text {jsep }}$ can be determined from a particle balance on the core plasma. In order to determine $\Gamma_{\text {jsep }}$ for the various impurity charge states it would be necessary to calculate the sputtering rate and transport of impurities in the divertor, edge and core regions, which is beyond the present state-of-the-art. Rather than introducing ambiguity by using approximate models to calculate $\Gamma_{\text {jsep }}$ for the impurities, we will assume a fixed impurity fraction, $\mathrm{f}_{\mathrm{z}}$, and impurity charge state, $\mathrm{Z}$, in the edge region. This allows Eq. (35) for the main ion species to be written

$$
\frac{d \Gamma_{i}}{d x}=n v_{i o n j}\left(1+f_{Z} Z\right) \quad, \quad \Gamma_{i}\left(x_{\text {sep }}\right)=\Gamma_{\text {isep }}
$$

where ' $\mathrm{i}$ ' and ' $\mathrm{z}$ ' now refer to the main ion and impurity species, respectively.

With this "constant impurity fraction" approximation, Eqs. (18)-(20) for the main ion species can be reduced to a simple pinch-diffusion flux relation

$\Gamma_{i}=-D_{i} \frac{\partial n_{i}}{\partial x}+n_{i}\left(D_{i} L_{T}^{-1}+v_{p i}\right), n_{i}\left(x_{s e p}\right)=n_{i s e p}$

where an effective ion diffusion coefficient has been defined

$$
D_{i} \equiv D_{i i}-f_{Z} D_{i Z}=\frac{m_{i} T_{i} v_{i Z}}{\left(e_{i} B_{\theta}\right)^{2}}\left[\left(1+\frac{\dot{v}_{d i}^{*}}{v_{i Z}}\right)-\frac{1}{Z}\right]
$$

and the temperature gradient scale length, $\mathrm{L}_{\mathrm{T}} \equiv-\mathrm{T} / \mathrm{dT} / \mathrm{dr}$ ), is assumed to be known for the moment (we plan to return to a similar determination of this quantity in a subsequent paper).

Equations (36) and (37) are coupled equations that can be solved for $\Gamma_{\mathrm{i}}(\mathrm{x})$ and $\mathrm{n}_{\mathrm{j}}(\mathrm{x})$, with the boundary conditions $\Gamma_{\mathrm{j}}\left(\mathrm{x}_{\mathrm{sep}}\right)=\Gamma_{\text {jsep }}$ and $\mathrm{n}_{\mathrm{i}}\left(\mathrm{x}_{\text {sep }}\right)=\mathrm{n}_{\text {isep. }}$. We will determine $\mathrm{n}_{\text {isep }}$ with a "2point" divertor model calculation, using plasma core power and particle balances to determine the heat and particle fluxes into the divertor-scrapeoff layer from the core ${ }^{24}$. The equations of the previous section will be solved to determine the poloidal rotation velocities, the radial electric field and the gyroviscous cross-field momentum transport frequency. 


\section{Neutral Penetration}

The interface current balance method ${ }^{23}$ is used to calculate the inward transport of a partial current, $\mathrm{J}_{\text {sep }}^{+}$, of neutral particles incident on the core plasma from the scrape-off layer at the separatrix. Defining the albedo as the ratio of inward to outward partial currents, $\alpha_{n} \equiv J_{n}^{+} / J_{n}^{-}$, a recursive relation relates the albedos at successive interfaces $n=1,2, \ldots N$ numbered successively from the separatrix $(n=1)$ inward to the innermost interface $(n=N)$.

$\alpha_{n}=\frac{\alpha_{n-1}\left(T_{n-1}^{2}-R_{n-1}^{2}\right)+R_{n-1}}{1-\alpha_{n-1} R_{n-1}}, \quad \mathrm{n}=2,3, \ldots \mathrm{N}$

Once the albedos are calculated by sweeping inward from $n=2$ to $n=N$, the ratio of outward partial currents at successive interfaces can be calculated by sweeping outward from $n=\mathrm{N}-1$ to $\mathrm{n}=1$ using the recursive relation

$\frac{J_{n}}{J_{n+1}}=R_{n} T_{n}^{-1} \alpha_{n+1}\left(T_{n}-R_{n}^{2} T_{n}^{-1}\right), \quad n=N-1, N-2, \ldots .1$

The appropriate boundary conditions are $\mathrm{J}^{+}{ }_{1}=\mathrm{J}_{\text {sep }}^{+}$and $\alpha_{\mathrm{N}}=\alpha_{\text {plasma. }}$. The quantity $\alpha_{\text {plasma }}$ is the albedo of a semi-infinite plasma medium, but the actual value is not important if the location of interface $\mathrm{N}$ is sufficiently far (several mean free paths) inside the separatrix that the neutral influx is highly attenuated. The quantities $R_{n}$ and $T_{n}$ are the reflection and transmission coefficients for the region of thickness $\Delta_{n}=x_{n+1}-x_{n}$ with total (ionization+chargeexchange+elastic scattering) mean-free-path $\lambda_{n}$ calculated for the local ion and electron temperature and assuming the neutrals to have the same local temperature as the plasma ions

$$
R_{n}=\frac{\frac{1}{2} C\left[\frac{\lambda_{n}}{\Delta_{n}}\right]\left[\frac{1}{2}-E_{3}\left(\frac{\lambda_{n}}{\Delta_{n}}\right)\right]\left[1-E_{2}\left(\frac{\lambda_{n}}{\Delta_{n}}\right)\right]}{1-C\left[1-\left[\frac{\lambda_{n}}{\Delta_{n}}\right]\left[\frac{1}{2}-E_{3}\left(\frac{\lambda_{n}}{\Delta_{n}}\right)\right]\right]}
$$




$$
T_{n}=E_{2}\left(\frac{\lambda_{n}}{\Delta_{n}}\right)+R_{n}
$$

where ' $c$ ' is the ratio of the charge-exchange plus elastic scattering cross sections to the total cross section, and $E_{m}(y)$ is the exponential integral function of $m$-th order and of argument ' $y$ '. The neutral density in each mesh interval is determined by equating the divergence of the neutral current to the ionization rate.

The transmission of uncollided 'cold' neutrals into the edge plasma is calculated from $J^{c}{ }_{n+1}=E_{2}\left(\Delta_{n} / \lambda_{n}^{c}\right) J_{n}^{c}$, where the mean-free-path $\lambda^{c}$ is calculated for the temperature of neutrals entering the scrape-off layer from the plenum region.

The incident partial current, $\mathrm{J}^{+}$sep, boundary condition is obtained from a $2 \mathrm{D}$ neutral transport calculation of ions incident on the divertor plate recycling as neutrals and returning through the divertor region and across the scrape-off layer to the separatrix ${ }^{24}$.

This coupled 2D neutral divertor transport and core penetration calculation is iterated to consistency with a core power and particle balance calculation and with a "2-point" divertor calculation $^{24}$. The overall neutral calculation procedure has been benchmarked by comparison with neutral density measurements inside the separatrix in DIII-D and with Monte Carlo calculations in Ref. 30, where further details of the calculation procedure and of the atomic data can be found.

\section{CALCULATIONS FOR A DIII-D H-MODE DISCHARGE}

A calculation with parameters representative of a lower single null divertor DIII-D Hmode plasma was made in order to investigate the extent to which the calculation model of this paper, which is based on ion particle and momentum balance and neutral particle recycling, predicts the observed structure and other features of the edge density pedestal. We modeled the DIII-D plasma as an effective circular plasma, but explicitly modeled the divertor geometry for the neutral recycling calculation and used a value $\xi=1$ for deuterium to represent the strong updown asymmetry in the ionization source term (see Eq. (16)). The representative discharge conditions (\#92976@3.2 s) used in the calculations were characterized by the parameters 
$\left[R=1.7 \mathrm{~m}, \quad \mathrm{a}=0.6 \mathrm{~m}, \quad \kappa=1.8, \quad I=1 \mathrm{MA}, \quad \mathrm{B}=2.1 \mathrm{~T}, \mathrm{P}_{\mathrm{nb}}=5 \mathrm{MW}, \mathrm{n}_{\mathrm{av}}=5.9 \mathrm{e} 19 / \mathrm{m}^{-3}, \mathrm{n}_{\mathrm{ped}}=4.9 \mathrm{e} 19 / \mathrm{m}^{-3}\right.$, $\mathrm{n}_{\text {sep }}=1.9 \mathrm{e} 19, \mathrm{~T}_{\text {eped }}=275 \mathrm{eV}, \mathrm{T}_{\text {iped }}=375 \mathrm{eV}, \mathrm{T}_{\text {esep }}=50 \mathrm{eV}, \mathrm{T}_{\text {isep }}=150 \mathrm{eV}$, deuterium plasma with edge carbon concentration $2.5 \%$ ]

The discharge was modeled, as described in Refs. 23 , in order to calculate the poloidally averaged recycling neutral flux flowing inward across the separatrix and the poloidally averaged ion flux flowing outward across the separatrix. These quantities and the measured (and calculated) plasma ion density at the separatrix, $\mathrm{n}_{\text {sep }}$, were then used as the boundary conditions for the calculation of the ion and neutral density profiles (and the profiles of radial electric field, poloidal rotation velocity, pinch velocity, etc.) in the edge plasma that was described in the previous section. Although the calculation model of the previous section yielded toroidal rotation velocities comparable to the measured values in the edge, we elected to use the measured velocities in Eqs. (28) to calculate the poloidal rotation velocities (the calculation of the poloidal rotation velocity was relatively insensitive to the value of the toroidal rotation velocity).

The plasma ion and neutral atom profiles in the edge plasma are shown in Fig. 1. An average inward recycling neutral flux across the separatrix of $5.32 \times 10^{20} \mathrm{at} / \mathrm{m}^{2}-\mathrm{s}$ was calculated. We observe that ion profile exhibits an edge pedestal structure.

In order to gain physical insight into the factors which cause the pedestal structure of the edge density profile in these calculations, it is useful to rewrite Eq. (37) in a form

$L_{n i}^{-1} \equiv-\frac{1}{n_{i}} \frac{d n_{i}}{d x}=\frac{\left(\Gamma_{i} / n_{i}-v_{p i}\right)}{D_{i}}-L_{T i}^{-1}$

that displays the dependence of the local density gradient scale length on the local ion particle radial velocity, $\mathrm{v}_{\mathrm{r}}=\Gamma_{\mathrm{i}} / \mathrm{n}_{\mathrm{i}}$, on the local value of the effective diffusion coefficient of Eq. (38), and on the local value of the radial pinch velocity of Eq. (20). We note that the local ion temperature gradient scale length also appears in Eqs. (37) and (42), implying a relationship between the ion temperature and density gradients in the edge. We plan to explore this relationship in a future paper, but for this paper we set the ion temperature gradient scale length to a large value, which has the effect of making the density gradient scale lengths calculated from Eqs. (37) or (42) 
somewhat smaller than they would otherwise be if the temperature gradient scale length was taken into account. Equation (42) was used to evaluate the pressure gradient term $\mathrm{P}_{\mathbf{j}}$ ' used to calculate the radial electric field in Eq. (34), and elsewhere.

The terms determining the density gradient scale length, hence the density profile, in Eq. (42) are plotted in Fig. 2. The radial velocity, $v_{r}=\Gamma_{i} / n_{i}$, exhibits a pronounced peaking as the separatrix is approached from the inside, which is due to an increase with radius (by a factor of 4 over the $5 \mathrm{~cm}$ range inside the separatrix) in $\Gamma_{\mathrm{i}}$ due to ionization of recycling neutrals and to the sharp decrease in $n_{i}$ just inside the separatrix. This increase in $v_{r}$ as the separatrix is approached from inside produces a sharp increase in magnitude of the negative poloidal velocity [first term on the right in Eq.(28)], as may be seen in Fig. 3. (The carbon poloidal velocity profile was calculated to be similar to, but of slightly larger negative magnitude than, the deuterium poloidal velocity profile shown in Fig. 3.) The radial electric field of Eq. (34) becomes strongly negative as the separatrix is approached because of the strong negative pressure gradient produced by the sharp decrease in $n_{i}$, as may be seen in Fig. 3. We note that the $v_{\theta}$ and $E_{r}$ profiles of Fig. 3 are characteristic of observed profiles in the edge pedestals of $\mathrm{H}$-mode plasmas.

The inward pinch velocity given by Eq. (20) and plotted in Fig. 2 was primarily determined by the toroidal electric field $\left(\mathrm{E}_{\varphi}\right)$ and friction $\left(v_{\mathrm{iz}}\right)$ terms well inside the separatrix. However, the momentum drag $\left(v^{*}{ }_{\mathrm{di}}\right)$ term involving the radial electric field and the poloidal rotation velocity became dominant and caused the sharp inward (negative) spike near the separatrix where both $v_{\theta}$ and $E_{r}$ became large and negative. This large inward pinch velocity in the edge pedestal, predominatly caused by the large negative values of $v_{\theta}$ and $E_{r}$, would seem to be the cause of the particle 'transport barrier' observed in the edge pedestal, at least for this discharge.

The diffusion coefficient of Eq. (38) is plotted in Fig. 4. The sharp drop just inside the separatrix is due to a drop in $v_{\mathrm{iz}}$, caused by a drop in $\mathrm{n}_{\mathrm{z}}$ (assumed in this model to be a constant fraction of $n_{i}$ ) relative to the values at locations further inside the separatrix. (Another calculation using a constant value of $\mathrm{n}_{\mathrm{z}}$ at all locations resulted in the same type of pedestal structure in the ion density and large negative spikes in $v_{\theta}$ and $E_{r}$ in the pedestal but not in a reduction in $D_{i}$ in the pedestal region.) The increase in $D_{i}$ at the separatrix was produced by an increase in the calculated value of $v^{*}{ }_{\mathrm{di}} / v_{\mathrm{iz}}$ at the separatrix relative to the value slightly inside the separatrix [see Eq. (38)], shown in Fig. 5. 
The profiles of the interspecies collision frequency, $v_{\mathrm{iz}}$, the gyroviscous momentum transport frequency, $v_{\mathrm{di}}$, the atomic (charge-exchange + elastic scattering + ionization) momentum exchange frequency, $v_{\mathrm{ati}}$, and the total momentum 'drag' frequency, $v^{*}{ }_{\mathrm{di}}=v_{\mathrm{di}}+v_{\mathrm{ati}}$, are plotted in Fig. 5. The atomic momentum exchange frequency naturally decreased with distance inside the separatrix because of attenuation of the neutrals. The gyroviscous momentum transport frequency also decreased with distance inside the separatrix because of the decreasing value of $L_{n}{ }^{-1}$ [used in evaluating the factor $G$ of Eq. (26)] and because of the decreasing values of $\mathrm{v}_{\theta}$ [used in evaluating the factor $\theta$ of Eq. (25)].

The calculated pedestal ion density shown in Fig. 1 is comparable to the measured electron density value of $4.2 \times 10^{19} / \mathrm{m}^{3}$. When the calculated plasma ion density gradient scale length calculated from Eq. (42) neglecting $\mathrm{L}_{\mathrm{Ti}}$ is corrected using the experimental value of $\mathrm{L}_{\mathrm{Ti}}$ in Eq. (42), the resulting $L_{n}=6 \mathrm{~cm}$ is comparable to the experimental value of $6.4 \mathrm{~cm}$ (see appendix of Ref. 15 for discussion of mapping measured gradient scale lengths to average values for the effective cylindrical model). The average radial electric field observed experimentally in the pedestal (steep gradient) region was $-13 \mathrm{kV} / \mathrm{m}$, which is comparable to the average calculated value shown in Fig. 3. We intend to make a more comprehensive comparison with experiment in the future.

\section{DISCUSSION}

We have formulated from plasma ion momentum and particle balance and neutral atom transport theory a 'first-principles' model for the self-consistent calculation of the edge density pedestal structure--radial profiles of plasma ion and neutral densities, radial electric field, poloidal and toroidal velocities, radial pinch velocity, and related quantities in the plasma edge of tokamaks.

We have found that these equations have a self-consistent solution in the plasma edge which exhibits an ion density pedestal and sharp negative spikes in the radial electric field, the poloidal rotation velocity and the pinch velocity in the pedestal region, for a calculation using the parameters of a DIII-D H-mode discharge. The mechanism for the creation of an edge (particle) transport barrier was found to be the creation of a large inward pinch velocity caused primarily by the large negative spikes in the radial electric field and poloidal velocity. These large 
negative spikes in the radial electric field and poloidal velocity in turn were caused primarily by the large pressure gradient and the large radially outward particle velocity required to satisfy the continuity condition as the ion density decreased sharply in the edge pedestal, respectively. An edge convective energy transport barrier is associated with an edge particle transport barrier, of course.

The results of this paper are not inconsistent with recent studies ${ }^{14-16}$ that demonstrated that the width of the edge density pedestal was comparable to the ionization mean-free-path (or the somewhat shorter transport mean-free-path that governs neutral penetration when chargeexchange and elastic scattering are taken into account) over a wide range of edge plasma conditions in DIII-D. However, in view of the new result of this paper on the dominant importance of a strong inward pinch term in determining particle transport in the plasma edge, the adequacy of the purely diffusive model of Ref. 31 (which omitted a pinch term) for interpretation $^{14-15}$ of these experimental data is questionable. The presence of recycling neutrals in the edge pedestal may be necessary in order for solutions to the coupled set of equations required by momentum and particle balance to have a pedestal structure, but this remains to be seen.

The edge plasma calculation models presented in this paper were derived from first principles-- the model for calculation of the plasma ion profile from particle and momentum conservation requirements, and the model for calculating the neutral atom profile from neutral particle transport theory. However, it was necessary to invoke further theory to evaluate the collisional friction and viscous momentum transfer terms-a Lorentz model was used for the friction and neoclassical models were used for the parallel and cross-field viscous force terms. To this extent, the overall model can be considered neoclassical. However, if any other viscous models were used to evaluate $\eta_{0 \mathrm{i}}$ and $v_{\mathrm{di}}$, the calculation model would be unchanged, but the results would differ to the extent that the other viscosity model yielded different values for $\eta_{0 \mathrm{i}}$ and $v_{\mathrm{di}}$.

There was nothing in the formulation of the calculation model of this paper that distinguished between $\mathrm{H}$-mode and L-mode plasmas, and in fact observed edge density gradients differ more quantitatively than qualitatively between the two regimes ${ }^{15}$. However, the large negative spikes in radial electric field and poloidal velocity found in the edge pedestal for the $\mathrm{H}$ mode discharge parameters used in the calculations of this paper (and presumably also the large 
inward pinch velocity) seem to be observed only in $\mathrm{H}$-mode plasmas. We conjecture that these distinctive $\mathrm{H}$-mode features will be predicted by the model of this paper for certain ranges of edge and plasma parameters, but not for others. We intend to investigate this conjecture in the near future. 


\section{REFERENCES}

1. A. E. Hubbard, "Physics and Scaling of the H-Mode Pedestal", Plasma Phys.Control. Fusion, 42, A15 (2000).

2. Y. Kamada, K. Ushigusa, T. Hatae, et al.,Plasma Phys. Control. Fusion, 36, A123 (1994) and 40, 1073 (1998).

3. R. J. Groebner and T. H. Osborne, "Scaling Studies of the High Mode Pedestal", Phys. Plasmas, 5, 1800 (1998).

4. T. H. Osborne, R. J. Groebner, L. L. Lao, et al.,Plasma Phys. Control. Fusion, 40, 131 (1998).

5. R. J. Groebner, D. M. Thomas and R. D. Deraian, "Evidence for Edge Gradients as Control Parameters of the Spontaneous High-Mode Transition", Phys. Plasmas, 8, 2722 (2001).

6. T. Onjun, G. Bateman, A. H. Kritz and G. Hammett, "Models for the Pedestal Temperature at the Edge of H-Mode Tokamak Plasmas", Phys. Plasmas, 9, 5018 (2002).

7. R. L. Miller, Y. R. Lin-Liu, T. H. Osborne and T. S. Taylor, Plasma Phys. Control. Fusion, 40, 753 (1998).

8. H. R. Wilson and R. L. Miller, Phys. Plasmas, 6, 873 (1999).

9. J. R. Ferron, M. S. Chu, G. L. Jackson, et al., "Modification of High-Mode Pedestal Instabilities in the DIII-D Tokamak", Phys. Plasmas, 7, 1976 (2000).

10. P. B. Snyder, H. R. Wilson, J. R. Ferron, et al., "Edge Localized Modes and the Pedestal: A Model Based on Coupled Peeling-Ballooning Modes”, Phys. Plasmas, 9, 2037 (2002).

11. P. B. Snyder, H. R. Wilson, J. R. Ferron, et al., Nucl. Fusion, 44, 320 (2004).

12. B. N. Rogers and J. F. Drake, Phys. Plasmas, 6, 2797 (1999).

13. F. L. Hinton and G. M. Staebler, Phys. Fluids B, 5, 1281 (1993).

14. R. J. Groebner, M. A. Mahdavi, A. W. Leonard, et al., "The Role of Neutrals in HighMode (H-Mode) Pedestal Formation", Phys. Plasmas, 9,2134 (2002).

15. R. J. Groebner, M. A. Mahdavi, A. W. Leonard, et al.,Nucl. Fusion, 44,204 (2004).

16. W. M. Stacey and R. J. Groebner, "A Framework for the Development and Testing of an Edge Pedestal Model: Formulation and Initial Comparison with DIII-D Data", Phys. Plasmas, 10, 2412 (2003). 
17. K-C Shaing, E. C. Crume and W. A. Houlberg, Phys. Fluids B, 2, 1492 (1990).

18. W. M. Stacey, "An Edge Pedestal Model Based on Transport and Atomic Physics", Phys. Plasmas, 8, 4073 (2001).

19. D. P. Coster, X. Bonnin, K. Borass, et al., Proc. $18^{\text {th }}$ Fusion Energy Conf., Sorrento, Italy, 2000 (IAEA, Vienna, 2001), IAEA-CSP-8/C, FEC_2000/pdf/exp4_20.pdf.

20. W. M. Stacey, "Investigation of Transport in the DIII-D Edge Pedestal", Phys. Plasmas, submitted (2003).

21. W. M. Stacey, "Particle Transport and Density Gradient Scale Lengths in the Edge Pedestal", Contrib. Plasma Phys., to be published (2004).

22. W. M. Stacey, Phys. Plasmas, 9, 3874 (2002).

23. W. M. Stacey, Phys. Plasmas, 4, 179 (1997).

24. W. M. Stacey, Phys. Plasmas, 5, 1015 (1998); 5, 3656 (1998); 8, 3673 (2001).

25. W. M. Stacey and D. J. Sigmar, Phys. Fluids, 28, 2800 (1985).

26. W. M. Stacey, A. W. Bailey, D. J. Sigmar and K. C. Shaing, Nucl. Fusion, 25, 463 (1985).

27. H. A. Claassen, H. Gerhauser and A. Rogister, Phys. Plasmas, 7, 3699 (2000).

28. A. N. Simakov and P. J. Catto, "Drift-Ordered Fluid Equations for Modeling Collisional Edge Plasma", Contr. Plasma Phys., to be published (2004).

29. W. M. Stacey and J. Mandrekas, Phys. Plasmas, 9, 1622 (2002).

30. W. M. Stacey, Nucl. Fusion, 40, 965 (2000).

31. M. A. Mahdavi, T. H. Osborne, A. W. Leonard, et al., Nucl. Fusion, 42, 52 (2002). 


\section{FIGURE TITLES}

1.Calculated plasma ion and neutral atom densities as a function of distance inside the separatrix for a DIII-D H-mode discharge.

2. Calculated outward radial ion total velocity and inward ion pinch velocity as a function of distance inside the separatrix for a DIII-D H-mode discharge.

3.Calculated radial electric field and deuterium ion poloidal rotation velocity as a function of distance inside the separatrix for a DIII-D H-mode discharge.

4.Effective deuterium ion diffusion coefficient as a function of distance inside the separatrix for a DIII-D H-mode discharge.

5.Momentum transfer frequencies as a function of distance inside the separatrix for a DIII-D H-mode discharge. 